\title{
Relación entre la edad cronológica y la mineralización del tercer molar inferior según método de Demirjian
}

\author{
Relationship between chronological age and mineralization of the lower third molar according to Demirjian stages
}

Milushka Miroslava Quezada Marquez ${ }^{1, a, b}$, Jorge Arturo Beltrán Silva ${ }^{1, a, b}$, Juan Benjamín Bernal Morales ${ }^{2, c}$, Alexis Evangelista Alva ${ }^{3, b}$, César Eduardo del Castillo López ${ }^{3, b}$

\section{RESUMEN}

Objetivos: Relacionar la edad cronológica y la mineralización del tercer molar inferior según los estadios de Demirjian en radiografías panorámicas digitales. Material y Métodos: Se realizó un análisis retrospectivo evaluando 1176 radiografías panorámicas digitales del Servicio de Radiología de la Clínica Dental de la Facultad de Estomatología, Universidad Peruana Cayetano Heredia, entre los años 2011 y 2012. Los casos tenían entre 7 23 años de edad. Resultados: En la pieza 38, el estadio D presentó una edad promedio de 14,05 $\pm 1,35$ años para el sexo femenino y 13,42 $\pm 1,30$ años para el sexo masculino. El tercer molar inferior en el sexo masculino presentó una maduración ligeramente anterior que el sexo femenino, esta diferencia fue estadísticamente significativa en los estadios D, E, F, G y H. No se encontró diferencia entre los estadios de Demirjian de la pieza 38 y 48 . En el estadio $\mathrm{H}$ el 100\% del sexo femenino y el 99,1\% del sexo masculino tuvieron 18 o más años. Conclusiones: Los resultados sugieren que el método de Demirjian, para evaluar la mineralización del tercer molar, puede ser una herramienta útil en el estudio de la edad cronológica.

PALABRAS CLAVE: Tercer molar, calcificación de dientes, odontología forense, determinación de la edad por los dientes. (Fuente: DeCS BIREME)

\section{SUMMARY}

Objectives: To relate the chronological age and the mineralization of the lower third molar according to Demirjian stages on digital panoramic radiographs. Methods: A retrospective analysis was conducted, evaluating 1176 digital panoramic radiographs from the Dental Clinic radiology area of the School of Dentistry at Universidad Peruana Cayetano Heredia, between 2011 and 2012. The cases were aged 7-23 years. Results: In tooth 38, the stage D represented an average age of $14.05 \pm 1.35$ years for females and $13.42 \pm 1.30$ years for males. The third

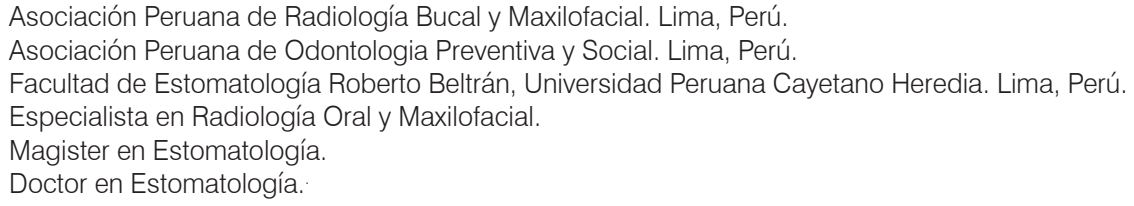


molar presented a slightly earlier maturation in males than in females, this difference was statistically significant in stages $D, E, F, G$ and $H$. No difference was found between the stages of Demirjian in teeth 38 and 48 . In stage H 100\% female and $99.1 \%$ male had 18 or more years. Conclusions: The results suggest that using Demirjian stages to assess the mineralization of third molar can be a useful tool in chronological age study.

KEYWORDS: Third molar, dental calcification, forensic dentistry, age determination by teeth. (Source: MeSH NLM)

\section{INTRODUCCIÓN}

La estimación de la edad juega un rol importante tanto para las áreas de endocrinología, pediatría, ortodoncia, antropología; así como para personas en estado de abandono y en casos de procesos médico-legales (penales y civiles) $(1,2)$. Durante el crecimiento de la persona ocurren cambios biológicos que pueden ser medibles, en la actualidad existe una gran cantidad de métodos adecuados, éstos incluyen: examen físico con determinación de las medidas antropométricas (talla y peso corporal), la inspección de los signos de maduración sexual, la edad basada en exámenes de rayos $\mathrm{X}$ de la mano izquierda $\mathrm{y}$ exámenes dentales de los estadios de erupción dentaria y grado de mineralización dental a través de las radiografías. Si el desarrollo de los huesos de la mano está completo, se debe evaluar las clavículas por medio radiográfico $(1,3)$.

La mineralización de los dientes abarca un rango de edad mucho más amplio que la erupción dental, además el grado de mineralización se puede medir radiográficamente siendo por lo tanto un método no invasivo (4). Los dientes en desarrollo son fiables en la estimación de la edad; las estructuras dentales mineralizadas son la parte más indestructible del cuerpo y exhiben el menor cambio de la estructura original, no sólo se mantienen hasta a la muerte, sino que también se conservan relativamente sin cambios por muchos miles de años (3). La mineralización del germen dentario se mantiene casi normal incluso frente a cambios nutricionales, hormonales $y$ patológicos (3).

La mineralización de los dientes evoluciona en secuencia invariable desde las puntas de las cúspides, luego a través de la corona con el esmalte y la dentina, a la formación de la unión cemento-adamantina. Una vez que la corona se ha formado, el desarrollo de la raíz avanza, terminando con el cierre del ápice de la raíz o raíces (5-7).Varios investigadores han ideado sistemas o métodos para medir la formación del diente y relacionarlo con la edad cronológica (2-4). Entre los métodos radiológicos utilizados en dentición permanente, en zona de molares, tenemos algunas clasificaciones que pueden ser descripciones verbales, descripciones gráficas, o ambas (5-11).

El método de Demirjian (7) ha sido aplicado a una variedad de poblaciones y los resultados han sido variados (12-19). Aunque el tercer molar es el diente más variable en la dentición con respecto a la cronología del desarrollo, a veces se utiliza para estimar la edad durante la adolescencia tardía y principios de la adultez $(20,21)$. Podría decirse que no hay otro indicador biológico más confiable disponible durante este periodo (14-21años); además el desarrollo del tercer molar es fácilmente evaluable a partir de radiografías dentales $(16,22)$.

En gran parte de los países, los 18 años marca la edad adulta o mayoría de edad y las implicancias legales cambian significativamente, es por ello que recientemente, la cronología del desarrollo del tercer molar se ha utilizado para juzgar si una persona es un menor de edad o un adulto $(17,19,22)$. Es importante mencionar que varios estudios han indicado que existen variaciones de acuerdo al grupo poblacional, país o etnia (12-19,23-32).

El año 2005 Olze y col. (13) realizaron un estudio de validación en 420 radiografías panorámicas evaluando el estado o grado de mineralización de los terceros molares inferiores, utilizaron cinco métodos diferentes que incluían las clasificaciones de Gleiser y Hunt (9), Demirjian y col. (7), Gustafson y Koch (8), Harris y Nortjè (10) y Kullman y col. (11). Los autores concluyeron que el método de Demirjian y col. (7) debería ser usado para evaluar la mineralización del tercer molar y estimar la edad en áreas forenses (13).

El objetivo del presente trabajo fue relacionar la edad cronológica y la mineralización del tercer 
molar inferior según los estadios de Demirjian en radiografías digitales de la Clínica Dental de la Facultad de Estomatología Roberto Beltrán de la Universidad Peruana Cayetano Heredia (UPCH), durante el periodo enero 2011 a julio 2012.

\section{MATERIAL Y MÉTODOS}

Este es un estudio de tipo descriptivo, transversal y analítico. La selección de las radiografías panorámicas se realizó a través de un muestreo por conveniencia utilizando la totalidad de radiografías que cumplían con los criterios de selección, el número de radiografías panorámicas digitales fue de 1176 y fueron tomadas del Servicio de Radiología de la Clínica Dental de la UPCH, durante el periodo enero 2011- julio 2012. Se incluyeron radiografías de pacientes entre 7 y 23 años de edad que presentaban ambos terceros molares inferiores. Se excluyeron las radiografías panorámicas que tenían patología quística o tumoral obvia, terceros molares inferiores con anomalías dentarias o posición transversal, radiografías distorsionadas o de mala calidad diagnóstica.

\section{Variables y covariables}

Las variables tomadas en cuenta fueron:

- Estadio de Demirjian: Estadio evolutivo del grado de mineralización dental. La medición de esta variable se evaluó en radiografías panorámicas digitales en las piezas 38 y 48. El examen radiográfico se analizó a través de los 8 estadios de mineralización de Demirjian: A, B, C, D, E, F, G, H (7) (Figura 1) y la visualización de la cripta ósea sin mineralización en su interior, se definió como estadio " 0 ".

- Edad cronológica: Tiempo transcurrido desde el nacimiento del paciente hasta la toma de la radiografía panorámica. La medición de esta variable se realizó estableciendo la diferencia aritmética entre la fecha de la toma radiográfica y la fecha de nacimiento.

- Tercer molar: Último molar permanente en mineralizarse y ubicado por distal del segundo molar en los humanos. La medición de esta variable se realizó en la radiografía panorámica según el lado derecho o izquierdo y los valores fueron pieza 38 y 48.

La covariable considerada fue:

- Sexo: La medición de esta variable se obtuvo de la base de datos de radiografías digitales a través del software Sidexis XG (SIRONA).

La investigadora principal fue capacitada y calibrada por un radiólogo oral y maxilofacial con una experiencia mínima de 10 años. Dicha calibración fue desarrollada en 45 radiografías panorámicas digitales tomadas con el equipo radiográfico Orthophos XG5 (Sirona Dental Systems GmbH Fabrikstrabe 31, Bensheim, Alemania), de pacientes con edad comprendida entre 7 y 23 años de edad, se evaluó directamente desde la pantalla del computador (Lenovo All-in-One ThinkCentre M71z de 20 pulgadas). Los estadios encontrados se compararon con los del experto (interobservador) y con los de la investigadora (intraobservador), el valor de Kappa encontrado fue 0,975 y 0,95 respectivamente.

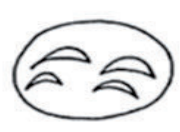

A

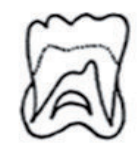

E

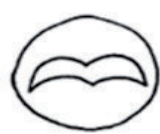

B

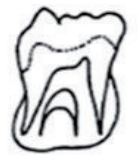

$\mathbf{F}$

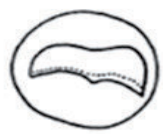

C

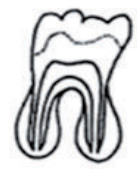

G

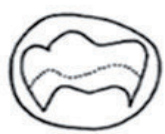

D

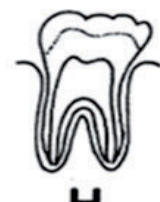

Figura 1. Estadios de formación de las terceras molares A-H, de Demirjian. Tomado de: Cantekin K, Yilmaz Y, Demirci T, Celikoglu M. Morphologic analysis of third-molar mineralization for eastern Turkish children and youth. J Forensic Sci. 2012; 57(2):531-4 (20). 
De forma similar que la calibración, se recolectaron los datos de: sexo, edad cronológica y estadio de Demirjian (7) en la pieza 38 y 48 en una ficha de recolección. Todos estos datos se evaluaron en radiografías digitales de las computadoras de la sala de informes radiográficos de la Clínica Dental, la cual es un área tranquila, libre de sonidos fuertes, con luz ambiental tenue y la evaluación de imágenes se realizó por un periodo no mayor de dos horas continuas por día.

Se estableció la distribución por sexo y según las diferentes edades, la edad cronológica según el estadio de Demirjian, sexo y lado del tercer molar e indicando el promedio, desviación estándar, valor mínimo y máximo. Luego se realizó la prueba de Kolmogorov-Smirnoff y Shapiro-Wilk para cada estadio de Demirjian y según sexo. La comparación de los estadios de Demirjian de ambos lados se realizó a través del porcentaje de acuerdo observado. La comparación entre los estadios de Demirjian según el sexo se realizó a través de la prueba $T$ student y $U$ de Mann-Witney con la finalidad de verificar si existían diferencias entre el sexo masculino y femenino. La correlación entre la edad cronológica y el estadio de mineralización se realizó a través del Coeficiente de Correlación Rho de Spearman, según sexo. Finalmente, se halló la probabilidad que una persona sea menor de edad o mayor de 18 años según el estadio de Demirjian. Se utilizó el paquete estadístico SPSS 18 para Windows. Los datos estadísticos del presente trabajo fueron llevados con un intervalo de confianza de $95 \%$ y un nivel de significancia de 0,05 .

\section{RESULTADOS}

De las 1176 radiografías, $679(57,7 \%)$ fueron de pacientes del sexo femenino y 497 (42,3\%) del sexo masculino. El promedio de edad para el grupo femenino fue $15,97( \pm 4,33)$ años y para el grupo masculino fue $15,74( \pm 4,36)$ años (Tabla 1$)$.

Tabla 1. Distribución según sexo y edad cronológica.

\begin{tabular}{|c|c|c|c|c|c|}
\hline \multirow{3}{*}{$\begin{array}{c}\text { Edad } \\
\text { cronológica } \\
\text { (años) }\end{array}$} & \multicolumn{4}{|c|}{ Sexo } & \multirow{3}{*}{ Total } \\
\hline & \multicolumn{2}{|c|}{ Femenino } & \multicolumn{2}{|c|}{ Masculino } & \\
\hline & $\mathbf{n}$ & $\%$ & $\mathbf{n}$ & $\%$ & \\
\hline $7,00-7,99$ & 7 & 0,6 & 11 & 0,9 & 18 \\
\hline $8,00-8,99$ & 24 & 2,0 & 19 & 1,6 & 43 \\
\hline $9,00-9,99$ & 29 & 2,5 & 25 & 2,1 & 54 \\
\hline $10,00-10,99$ & 36 & 3,1 & 36 & 3,1 & 72 \\
\hline $11,00-11,99$ & 47 & 4,0 & 22 & 1,9 & 69 \\
\hline $12,00-12,99$ & 50 & 4,3 & 33 & 2,8 & 83 \\
\hline $13,00-13,99$ & 65 & 5,5 & 38 & 3,2 & 103 \\
\hline $14,00-14,99$ & 45 & 3,8 & 35 & 3,0 & 80 \\
\hline $15,00-15,99$ & 52 & 4,4 & 36 & 3,1 & 88 \\
\hline $16,00-16,99$ & 44 & 3,7 & 44 & 3,7 & 88 \\
\hline $17,00-17,99$ & 39 & 3,3 & 34 & 2,9 & 73 \\
\hline $18,00-18,99$ & 38 & 3,2 & 34 & 2,9 & 72 \\
\hline $19,00-19,99$ & 49 & 4,2 & 30 & 2,6 & 79 \\
\hline $20,00-20,99$ & 40 & 3,4 & 26 & 2,2 & 66 \\
\hline $21,00-21,99$ & 46 & 3,9 & 25 & 2,1 & 71 \\
\hline $22,00-22,99$ & 39 & 3,3 & 31 & 2,6 & 70 \\
\hline $23,00-23,99$ & 29 & 2,5 & 18 & 1,5 & 47 \\
\hline Total & 679 & $\mathbf{5 7 , 7}$ & 497 & 42,3 & 1176 \\
\hline
\end{tabular}

Tabla 2. Distribución de estadios de Demirjian en la pieza 38 según sexo y edad cronológica.

\begin{tabular}{|c|c|c|c|c|c|c|c|c|c|c|c|}
\hline \multirow{2}{*}{$\begin{array}{c}\text { Estadio } \\
\text { Demirjian }\end{array}$} & \multirow{2}{*}{$\begin{array}{c}\text { Femenino } \\
\mathbf{N}\end{array}$} & \multicolumn{4}{|c|}{ Edad cronológica (años) } & \multirow{2}{*}{$\begin{array}{c}\text { Masculino } \\
\text { N }\end{array}$} & \multicolumn{4}{|c|}{ Edad cronológica (años) } & \multirow[b]{2}{*}{ Valor $\mathbf{p}$} \\
\hline & & Media & DE & Mínimo & Máximo & & Media & DE & Mínimo & Máximo & \\
\hline $\mathbf{0}$ & 11 & 8,68 & 1,11 & 7,00 & 10,97 & 13 & 8,55 & 0,88 & 7,21 & 9,88 & $0,751^{*}$ \\
\hline $\mathbf{A}$ & 25 & 8,92 & 1,14 & 7,46 & 11,74 & 26 & 8,66 & 1,03 & 7,00 & 10,77 & $1,000 * *$ \\
\hline B & 26 & 9,83 & 0,95 & 8,27 & 11,74 & 26 & 9,82 & 1,16 & 8,03 & 13,38 & $0,976^{*}$ \\
\hline $\mathbf{C}$ & 109 & 11,34 & 1,20 & 8,17 & 16,19 & 50 & 11,12 & 1,19 & 7,97 & 15,18 & $0,350 * *$ \\
\hline D & 120 & 14,05 & 1,35 & 11,38 & 19,40 & 74 & 13,42 & 1,30 & 10,88 & 17,48 & $0,014 * *$ \\
\hline $\mathbf{E}$ & 84 & 15,50 & 1,69 & 12,38 & 20,01 & 59 & 14,79 & 1,07 & 12,71 & 18,05 & $0,005^{*}$ \\
\hline $\mathbf{F}$ & 75 & 17,27 & 1,78 & 12,41 & 21,60 & 60 & 16,38 & 1,24 & 11,89 & 19,90 & $0,001 *$ \\
\hline $\mathbf{G}$ & 112 & 19,59 & 1,90 & 14,81 & 23,98 & 72 & 18,56 & 1,39 & 16,11 & 22,97 & $<0,001 * *$ \\
\hline $\mathbf{H}$ & 117 & 21,86 & 1,28 & 18,35 & 23,92 & 117 & 21,32 & 1,49 & 17,52 & 23,82 & $0,022 * *$ \\
\hline
\end{tabular}

$D E=$ desviación estándar

* prueba t de Student, ** prueba U de Mann-Whitney

Sexo femenino $r=0,939$ (Rho de Spearman)

Sexo masculino $r=0,957$ (Rho de Spearman) 
Al evaluar la pieza 38 , se encontró que el promedio de la edad cronológica (edad decimal) en pacientes del sexo femenino en el estadio de Demirjian D fue $14,05 \pm 1,35$ años y en el estadio $\mathrm{H}$ fue $21,86 \pm 1,28$ años. En pacientes del sexo masculino, en el estadio D fue $13,42 \pm 1,30$ años y en el estadio $H$ fue 21,32 $\pm 1,49$ años. Se encontró diferencias estadísticamente significativas, según sexo en los estadios D, E, F, G y $\mathrm{H}$ (Tabla 2), resultados similares se encontraron en la pieza 48 (Tabla 3 ).
Al comparar los estadios de Demirjian observados en las piezas 38 y 48 de cada radiografía, se encontró que los estadios coinciden en 89,54\% (en 1053 radiografías) del total de 1176 radiografías. Los casos de no coincidencia fue el 10,37\% (en 122 radiografias) con una diferencia no mayor o menor a un estadio, salvo en un caso $(0,09 \%)$ en que la diferencia fue de dos estadios. En ambas piezas, 38 y 48, los estadios con menor coincidencia corresponden a los estadios 0, A y B y los estadios con mayor coincidencia corresponden a los estadios C, D, E, F, G y H.

Tabla 3. Distribución de estadios de Demirjian en la pieza 48 según sexo y edad cronológica.

\begin{tabular}{|c|c|c|c|c|c|c|c|c|c|c|c|}
\hline \multirow{2}{*}{$\begin{array}{c}\text { Estadio } \\
\text { Demirjian }\end{array}$} & \multirow{2}{*}{$\begin{array}{c}\text { Femenino } \\
\mathbf{N}\end{array}$} & \multicolumn{4}{|c|}{ Edad cronológica (años) } & \multirow{2}{*}{$\begin{array}{c}\text { Masculino } \\
\mathbf{N}\end{array}$} & \multicolumn{4}{|c|}{ Edad cronológica (años) } & \multirow[b]{2}{*}{ Valor p } \\
\hline & & Media & DE & Mínimo & Máximo & & Media & DE & Mínimo & Máximo & \\
\hline $\mathbf{0}$ & 10 & 8,15 & 0,81 & 7,00 & 9,58 & 12 & 8,88 & 0,99 & 7,21 & 10,27 & $0,062 *$ \\
\hline $\mathbf{A}$ & 22 & 9,12 & 1,20 & 7,46 & 11,74 & 29 & 8,62 & 1,03 & 7,00 & 10,77 & $0,300 *$ \\
\hline B & 32 & 9,63 & 0,91 & 8,17 & 12,29 & 20 & 9,47 & 0,83 & 8,03 & 11,55 & $0,765^{*}$ \\
\hline $\mathbf{C}$ & 107 & 11,41 & 1,14 & 8,57 & 16,19 & 54 & 11,11 & 1,09 & 7,97 & 14,03 & $0,036^{* *}$ \\
\hline D & 122 & 13,98 & 1,31 & 11,05 & 19,40 & 72 & 13,48 & 1,40 & 10,88 & 17,48 & $0,005 * *$ \\
\hline $\mathbf{E}$ & 87 & 15,69 & 1,62 & 12,38 & 20,01 & 64 & 14,76 & 1,06 & 12,71 & 18,05 & $<0,001^{*}$ \\
\hline $\mathbf{F}$ & 73 & 17,37 & 1,80 & 12,41 & 21,60 & 61 & 16,46 & 1,24 & 11,89 & 19,90 & $0,001^{*}$ \\
\hline G & 115 & 19,76 & 1,93 & 14,81 & 23,67 & 75 & 18,79 & 1,47 & 16,11 & 22,97 & $<0,001 * *$ \\
\hline $\mathbf{H}$ & 111 & 21,84 & 1,34 & 18,35 & 23,98 & 110 & 21,39 & 1,47 & 17,52 & 23,82 & $0,028^{* *}$ \\
\hline
\end{tabular}

$D E=$ desviación estándar

* prueba $t$ de Student, ** prueba U de Mann-Whitney

Sexo femenino $r=0,940$ (Rho de Spearman)

Sexo masculino $r=0,955$ (Rho de Spearman)

Tabla 4. Probabilidad de ser menor o mayor de 18 años según sexo y estadio de Demirjian.

\begin{tabular}{lrcrc}
\hline & \multicolumn{2}{c}{ Pieza 38 } & \multicolumn{2}{c}{ Pieza 48 } \\
Estadio de & $\begin{array}{c}\text { Menor de 18 } \\
\text { años }\end{array}$ & $\begin{array}{c}\mathbf{1 8} \text { o más de } \\
\mathbf{1 8} \text { años }\end{array}$ & $\begin{array}{c}\text { Menor de } \\
\mathbf{1 8} \text { años }\end{array}$ & $\begin{array}{c}\text { 18 o más de 18 } \\
\text { años }\end{array}$ \\
\hline Femirjian & & & & \\
Femenino & $99,2 \%$ & $0,8 \%$ & $99,2 \%$ & $0,8 \%$ \\
Estadio D & $90,5 \%$ & $9,5 \%$ & $90,8 \%$ & $9,2 \%$ \\
Estadio E & $65,3 \%$ & $34,7 \%$ & $63,0 \%$ & $37,0 \%$ \\
Estadio F & $21,4 \%$ & $78,6 \%$ & $19,1 \%$ & $88,9 \%$ \\
Estadio G & $0,0 \%$ & $100,0 \%$ & $0,0 \%$ & $100,0 \%$ \\
Estadio H & & & & \\
Masculino & $100,0 \%$ & $0,0 \%$ & $100,0 \%$ & $0,0 \%$ \\
Estadio D & $98,3 \%$ & $1,7 \%$ & $98,4 \%$ & $1,6 \%$ \\
Estadio E & $93,3 \%$ & $6,7 \%$ & $93,5 \%$ & $6,5 \%$ \\
Estadio F & $40,3 \%$ & $59,7 \%$ & $33,3 \%$ & $66,7 \%$ \\
Estadio G & $0,8 \%$ & $99,2 \%$ & $0,9 \%$ & $99,1 \%$ \\
Estadio H & & & & \\
\hline
\end{tabular}


Al evaluar la probabilidad que un individuo sea menor de 18 años o mayor de edad (18 años o más), se encontró en el estadio $\mathrm{H}$, en el grupo del sexo femenino, que el $100 \%$ era mayor de edad; mientras que en el sexo masculino el 99,1\% lo era (Tabla 4).

\section{DISCUSIÓN}

Diversos estudios han demostrado que la mineralización de los dientes es un buen parámetro para estimar la edad biológica $(12,14,16,19,23,32)$. Para los niños, la estimación de la edad se puede llevar a cabo de acuerdo con las etapas de mineralización de siete dientes permanentes $(1,3)$. Sin embargo, para las personas durante la adolescencia tardía y la adultez temprana (es decir, de 14-18 años de edad), el tercer molar es el único diente que aún se encuentra en desarrollo.

Las etapas propuestas por Demirjian y col. (7) en 1973, constituyen la técnica más práctica y simple establecida hasta el momento y su método es uno de los más utilizados en todo el mundo $(2,4,12,13,15$ 19,29,32) debido a la elevada concordancia interobservador $(18,22,27,29,31)$; y además, la literatura señala que existe una fuerte correlación entre los estadios de Demirjian en el tercer molar y la edad cronológica $(14-19,27)$.

Algunos estudios similares al presente, evaluaron los cuatro terceros molares $(2,13,15-18,21,22-$ 24,26,28-30), otros solo los terceros molares inferiores $(4,14,27,31,32,38)$ y otros solo la pieza 38 (19,29). Kasper y col. (17), Mincer y col. (30) y Sisman y col. (27) utilizan de forma indistinta el tercer molar del lado derecho e izquierdo, de esta manera duplican el número de observaciones. El rango de edad de la selección de la muestra en los diferentes estudios es variable y ello depende de los objetivos. Algunos estudios van enfocados principalmente a estimar la mayoría de edad, entonces sus muestras se encuentra aproximadamente en el rango entre los 14 y 25 años $(2,12,14,17,19,22,23,25,26,30,32)$. Otros registran desde los inicios de la mineralización del tercer molar, por lo que el límite inferior se encuentra aproximadamente a los siete años de edad $(16,20,21,24,27,29,32)$ (Tabla 5).

\section{Distribución de la edad según sexo}

Algunos estudios reportan que en sus muestras, el grupo femenino fue ligeramente mayor en número comparado con el masculino $(7,12,14,15,17,19,21,25$ 27,32); mientras que otros reportan que el grupo masculino lo fue $(17,22,24,29)$. En el presente estudio el $57,7 \%$ de las radiografías fueron de pacientes de sexo femenino y el $42,3 \%$ pertenecieron al sexo masculino.

\section{Distribución de estadios de Demirjian según edad cronológica}

Hay evidencia que dependiendo el grupo étnico se observan diferencias en la edad cronológica según el grado de mineralización en el tercer molar inferior $(2,4,17,23)$, lo que significa que las etapas propuestas por Demirjian y col. (7) reflejan diferentes edades de una población a otra. Esto podría representar un problema importante para los investigadores $y$ forenses que intentan identificar a personas de diferentes países, de allí la necesidad de establecer un sistema de evaluación para diferentes poblaciones. En el presente estudio se encontró que el estadio 0 ya está presente desde los 7 años de edad, no descartamos la posibilidad que también se pueda observar en pacientes menores. En otros países, como Turquía, el inicio de la mineralización (estadio A) del tercer molar se describe en una edad promedio de 11 años (27), en China a los 10 años $(15,29)$, mientras que en Portugal, Brasil, $(21,32)$ y Perú aproximadamente a los 8,5 años de edad (Tabla 6).

A nivel mundial, según los artículos científicos revisados, se encontró una gran variabilidad en la edad cronológica en el estadio de mineralización de la corona completa. Así, en el sexo femenino la edad promedio fue: en Brasil de aproximadamente 13,2 años (32), en Portugal (21) y Turquía $(20,27)$ de 13,5 años, en Canadá (28) y China (15) de 14 años, en España (14) y Malasia (31) de 15 años, en EE.UU. $(17,30)$ de 15.5 años, en Alemania (2) de 15.8 años y en Italia (19) y Japón (22) de 16 años de edad. En el presente estudio la edad promedio fue de 14 años, es decir ligeramente mayor que la población de Brasil, Portugal y Turquía. En el sexo masculino el orden fue el siguiente, en Brasil (32), Portugal (21) Canadá (28) y Turquía $(20,27)$ de aproximadamente 13 años, en China (15) de 14 años, en Malasia (31) de 14,7 años, en España (14) de 15 años, en EE.UU. $(17,30)$ de 15,5 años y en Alemania (2), Italia (19) y Japón (22) de 16 años de edad. En el presente estudio la edad promedio fue de 13,4 años, es decir ligeramente 
Tabla 5. Rango de edad, número de casos y piezas evaluadas reportados en varias poblaciones.

\begin{tabular}{|c|c|c|c|c|c|}
\hline Autores & Año & País & Casos & Rango de Edad (años) & Piezas \\
\hline Quezada & 2013 & Perú & 1176 & $7-23$ & 38,48 \\
\hline De Olivera $^{32}$ & 2012 & Brasil & 407 & $6-25$ & 38,48 \\
\hline Medeiros $^{16}$ & 2010 & Brasil & 173 & $5.5-21$ & $18,28,38,48$ \\
\hline Solari $^{12}$ & 2002 & EEUU* & 679 & $14-25$ & $18,28,38,48$ \\
\hline Kasper $^{17}$ & 2009 & EEUU* & 950 & $12-22$ & $18,28,38,48$ \\
\hline Mincer $^{30}$ & 1993 & EEUU & 823 & $14.1-24.9$ & $18,28,38,48$ \\
\hline Blankenship $^{23}$ & 2007 & EEUU & 1200 & $14-24.9$ & $18,28,38,48$ \\
\hline $\mathrm{Olze}^{28}$ & 2010 & Canadá & 605 & $11-29$ & $18,28,38,48$ \\
\hline Prieto $^{14}$ & 2005 & España & 1054 & $14-21$ & 38,48 \\
\hline Cameriere $^{19}$ & 2008 & Italia & 906 & $14-23$ & 38 \\
\hline Caldas $^{21}$ & 2011 & Portugal & 737 & $6-22$ & $18,28,38,48$ \\
\hline Boonpitaksathit ${ }^{26}$ & 2011 & Inglaterra & 1223 & $12.6-24.9$ & $18,28,38,48$ \\
\hline Knell $^{4}$ & 2009 & Suiza & 1260 & $15-22$ & 38,48 \\
\hline $\mathrm{Olze}^{2}$ & 2004 & Alemania & 1430 & $12-26$ & $18,28,38,48$ \\
\hline Verma $^{25}$ & 2011 & India & 220 & $14-24$ & 38,48 \\
\hline $\mathrm{Rai}^{18}$ & 2010 & Irán & 1200 & $10-27$ & $18,28,38,48$ \\
\hline Olze $^{2}$ & 2004 & Japón & 1597 & $12-26$ & $18,28,38,48$ \\
\hline Arany ${ }^{22}$ & 2004 & Japón & 1282 & $14-24$ & $18,28,38,48$ \\
\hline $\operatorname{Lin}^{15}$ & 2010 & China & 3100 & $4.1-26.9$ & $18,28,38,48$ \\
\hline $\mathrm{Li}^{29}$ & 2012 & China & 2078 & $5-23$ & $18,28,38,48$ \\
\hline Sisman $^{27}$ & 2007 & Turquía & 900 & $8-25$ & 38,48 \\
\hline Cantekin $^{20}$ & 2012 & Turquía & 1348 & $7-22$ & 38 \\
\hline Johan $^{31}$ & 2011 & Malasia & 1080 & $14-25$ & 38,48 \\
\hline $\mathrm{Olze}^{2}$ & 2004 & Sudáfrica & 584 & $12-26$ & $18,28,38,48$ \\
\hline Hassan $^{24}$ & 2007 & Egipto & 380 & $8-26$ & $18,28,38,48$ \\
\hline
\end{tabular}

* población de hispanos

mayor que Brasil, Canadá, Portugal y Turquía (Tabla $6)$.

El final de la mineralización dental concluye con el cierre apical, en el sexo femenino se encontró que el promedio de edad en España fue aproximadamente 19 años (14), en Inglaterra (26) e hispanos residentes en EE.UU. (17) de 20 años, en EE.UU. de 21 años (30), en Brasil (32), Sudáfrica (2), Inglaterra (26) y Japón (22) de 22 años y en Canadá (28), Turquía (27) y Malasia (31) de 23 años de edad. En el presente estudio se encontró una edad promedio de 21,9 años. Este valor coincide con los encontrados en Brasil, probablemente porque somos países latinos; asimismo, en Japón y Sudáfrica el valor tambien es similar, quizas porque en el Perú existen colonias de inmigrantes y descendientes de estos países. En el sexo masculino se encontró que el promedio de la edad en EE.UU. (17,30), Italia (19) e Inglaterra (26) fue de 20 años, en Brasil (32), Japón (22) y China (29) de 21 años, en Alemania (2), China (15), Turquía (27), Malasia (31) y Sudáfrica (2) de 22 años y en el presente estudio la edad promedio fue de 21,3 años. En el sexo femenino la edad promedio fue 21,9 años, similar al promedio de Brasil, Japón y China (Tabla 6).

\section{Diferencia de edad entre el sexo femenino y masculino}

La mayoría de publicaciones revelan que sí existe diferencia en la mineralización del tercer molar entre hombres y mujeres, encontrándose que los hombres presentan cronológicamente un desarrollo mas temprano que las mujeres $(12,14,15,17,19,22,2$ $6,28,29,32)$. Arany y col. en Japón (22) encontraron que los hombres tuvieron un desarrollo más precoz que las mujeres en 8 meses. Kasper y col. (17) en un estudio realizado en una población hispana 
Tabla 6. Distribución de los estadios de Demirjian en el tercer molar según edad y sexo reportados en varias poblaciones.

\begin{tabular}{|c|c|c|c|c|c|c|c|c|c|c|c|c|c|c|c|c|c|c|c|}
\hline \multirow[t]{2}{*}{ País } & \multirow[t]{2}{*}{ Pieza } & \multicolumn{2}{|c|}{$\mathbf{0}$} & \multicolumn{2}{|c|}{$\mathbf{A}$} & \multicolumn{2}{|c|}{ B } & \multicolumn{2}{|c|}{$\mathrm{C}$} & \multicolumn{2}{|c|}{ D } & \multicolumn{2}{|c|}{$\mathbf{E}$} & \multicolumn{2}{|c|}{ F } & \multicolumn{2}{|c|}{$\mathbf{G}$} & \multicolumn{2}{|c|}{$\mathbf{H}$} \\
\hline & & $\mathbf{F}$ & $\mathbf{M}$ & $\mathbf{F}$ & $\mathbf{M}$ & $\mathbf{F}$ & $\mathbf{M}$ & $\mathbf{F}$ & M & F & $\mathbf{M}$ & $\mathbf{F}$ & $\mathbf{M}$ & $\mathbf{F}$ & $\mathbf{M}$ & $\mathbf{F}$ & $\mathbf{M}$ & F & $\mathbf{M}$ \\
\hline Perú, Quezada & 38 & 8,7 & 8,6 & 8,9 & 8,7 & 9,8 & 9,8 & 11,3 & 11,1 & 14,1 & 13,4 & 15,5 & 14,8 & 17,3 & 16,4 & 19,6 & 18,6 & 21,9 & 21,3 \\
\hline Perú, Quezada & 48 & 8,2 & 8,9 & 9,1 & 8,6 & 9,6 & 9,5 & 11,4 & 11,1 & 14,0 & 13,5 & 15,7 & 14,8 & 17,4 & 16,5 & 19,8 & 18,8 & 21,8 & 21,4 \\
\hline Brasi $^{13}$ & 38 & 7,7 & 7,8 & 8,2 & 8,5 & 10,1 & 9,5 & 10,7 & 10,9 & 13,2 & 12,9 & 14,0 & 14,7 & 16,6 & 15,9 & 18,4 & 17,3 & 21,6 & 21,7 \\
\hline Brasi $^{32}$ & 48 & 7,5 & 7,6 & 8,4 & 8,5 & 10,1 & 9,5 & 11,3 & 10,8 & 13,2 & 13,0 & 14,4 & 14,1 & 16,5 & 15,6 & 18,4 & 17,4 & 21,6 & 21,7 \\
\hline EEUU*17 & $38 / 48$ & - & - & - & - & - & - & 15,7 & 15,2 & 15,4 & 14,8 & 16,5 & 15,8 & 17,4 & 16,8 & 18,4 & 17,9 & 20,1 & 19,9 \\
\hline EEUU $^{30}$ & $38 / 48$ & - & - & - & - & - & - & & & 16,0 & 15,5 & 16,9 & 17,3 & 17,7 & 17,5 & 19,1 & 18,3 & 20,9 & 20,5 \\
\hline Canadá ${ }^{28}$ & 38 & - & - & - & - & - & - & 11,9 & 12,5 & 14,0 & 13,0 & 16,0 & 15,3 & 18,0 & 17,4 & 20,3 & 19,8 & 23,7 & 23,2 \\
\hline España $^{14}$ & 38 & - & - & - & - & - & - & 15,0 & 15,0 & 15,1 & 15,1 & 16,0 & 15,2 & 16,8 & 16,4 & 18,4 & 17,9 & 19,7 & 19,7 \\
\hline España $^{14}$ & 48 & - & - & - & - & - & - & 14,9 & 14,8 & 15,1 & 15,1 & 15,9 & 15,2 & 16,8 & 16,4 & 18,5 & 17,7 & 19,6 & 19,7 \\
\hline Italia $^{19}$ & 38 & - & - & - & - & - & - & 15,2 & 15,1 & 16,3 & 15,8 & 16,6 & 16,1 & 17,8 & 17,3 & 18,6 & 18,6 & 20,3 & 20,0 \\
\hline Portugal $^{21}$ & 38 & - & - & 8,4 & 8,3 & 9,3 & 9,6 & 11,5 & 11,5 & 14,0 & 13,1 & 16,3 & 14,7 & 16,5 & 16,6 & 18,1 & 18,4 & - & - \\
\hline Portugal $^{21}$ & 48 & - & - & 8,6 & 8,5 & 9,8 & 9,6 & 11,2 & 11.5 & 13,4 & 13,2 & 15,9 & 14,3 & 16,8 & 15,8 & 18,3 & 18,4 & - & - \\
\hline Inglaterra $^{26}$ & 38 & - & - & - & - & - & - & - & - & - & - & 15,5 & 15,3 & 16,7 & 16,5 & 18,3 & 17,6 & 20,3 & 19,5 \\
\hline Inglaterra $^{26}$ & 48 & - & - & - & - & - & - & - & - & - & - & 15,6 & 15,4 & 16,5 & 16,4 & 18,2 & 17,7 & 19,7 & 19,8 \\
\hline Alemania $^{2}$ & 48 & - & - & - & - & - & - & - & - & 15,8 & 16,1 & 17,2 & 16,7 & 19,0 & 18,2 & 21,6 & 21,2 & 22,9 & 22,5 \\
\hline Japón ${ }^{22}$ & 38 & - & - & - & - & - & - & 14,7 & 14,8 & 16,0 & 15,4 & 17,3 & 16,4 & 18,3 & 17,4 & 19,4 & 18,6 & 21,8 & 21,6 \\
\hline Japón ${ }^{22}$ & 48 & - & - & - & - & - & - & 14,8 & 14,7 & 16,1 & 15,9 & 17,2 & 16,3 & 18,1 & 17,2 & 19,4 & 18,7 & 21,8 & 21,6 \\
\hline China $^{29}$ & 38 & 8,7 & 7,7 & 10,3 & 9,4 & 10,8 & 10,9 & 12,5 & 12,6 & 14,4 & 14,3 & 16,5 & 16,5 & 17,9 & 18,0 & 19,9 & 19,2 & 21,6 & 21,3 \\
\hline China $^{29}$ & 48 & 8,4 & 8,3 & 10,0 & 9,8 & 10,7 & 10,5 & 12,4 & 12,5 & 14,6 & 14,2 & 16,5 & 16,7 & 18,2 & 18,0 & 19,8 & 19,4 & 21,7 & 21,2 \\
\hline China $^{15}$ & 38 & - & - & 10,3 & 9,8 & 11,1 & 11,1 & 12,6 & 12,6 & 13,9 & 13,5 & 15,8 & 15,3 & 17,9 & 17,1 & 20,6 & 19,3 & 23,3 & 22,7 \\
\hline China $^{26}$ & 48 & - & - & 10,3 & 9,9 & 11,1 & 10,9 & 12,6 & 12,6 & 13,9 & 13,5 & 15,8 & 15,1 & 17,9 & 17,1 & 20,6 & 19,3 & 23,3 & 22,7 \\
\hline Turquía $^{20}$ & 38 & - & - & 9,6 & 9,2 & 10,7 & 10,9 & 11,6 & 12,1 & 13,3 & 13,2 & 14,2 & 14,2 & 15,8 & 15,7 & 17,2 & 17,2 & 20,5 & 20,4 \\
\hline Turquía $^{27}$ & $38 / 48$ & - & - & 12,6 & 11,5 & 11,4 & 11,6 & 12,5 & 12,2 & 13,6 & 12,9 & 15,4 & 14,4 & 16,8 & 16,9 & 19,3 & 18,1 & 22,7 & 22,1 \\
\hline Malasia $^{31}$ & 38 & - & - & - & - & - & - & 14,6 & 14,5 & 15,2 & 14,8 & 16,0 & 15,8 & 17,8 & 17,0 & 20,2 & 19,0 & 22,6 & 22,4 \\
\hline Malasia $^{31}$ & 48 & - & - & - & - & - & - & 14,6 & 14,5 & 15,2 & 14,7 & 15,9 & 15,8 & 17.9 & 17,2 & 20,1 & 19,2 & 22,7 & 22,5 \\
\hline Sudáfrica $^{2}$ & 48 & - & - & - & - & - & - & - & - & 14,5 & 13,9 & 15,9 & 15,2 & 17,4 & 18,7 & 19,8 & 20,8 & 22,4 & 22,6 \\
\hline
\end{tabular}

(Texas) encontraron que los hombres desarrollan más rápido que las mujeres con una discrepancia de 8 a 18 meses; de Olivera y col.(32) encontraron que en Brasil también los hombres desarrollan de 1 a 13 meses antes que las mujeres; pero en el cierre apical, las mujeres se adelantan ligeramente a los hombres. En el presente estudio se encontró que los hombres peruanos estudiados tuvieron un desarrollo precoz o anterior del tercer molar entre 8 a 12 meses con respecto a las mujeres.

\section{Estadios de mineralización del lado derecho e izquierdo}

El presente estudio y otros consultados concuerdan que no existe diferencia entre el estadio de mineralización del tercer molar del lado derecho con el del lado izquierdo (14-16,22,32). Sin embargo, Mincer y col. (30) registraron que el porcentaje de concordancia solo alcanzó el 74\% . Por lo mencionado, podemos afirmar que para estimar la edad se puede utilizar cualquiera de las terceras molares inferiores o ambas.

\section{Determinación de la mayoría de edad según estadio H}

Gran parte de los estudios muestran que existe una probabilidad por encima del $97 \%$ en mujeres y $96 \%$ en hombres que, al cerrar el ápice (estadio $\mathrm{H}$ ), tengan al menos 18 años de edad $(19,21,22,24,31,32)$. Nuestros resultados muestran en el estadio $\mathrm{H}$ la probabilidad que un individuo tenga 18 años o más, es del $99,1 \%$ para el sexo masculino y $100 \%$ para el sexo femenino. Es de mencionar que en el grupo de hombres sólo hubo un caso que presentó cierre apical antes de los 18 años de edad (17,52 años) (Tabla 7). Sin embargo, algunos estudios revelan porcentajes menores para establecer mayoría de edad, según el cierre apical $(12,14,17,30,23)$. 
Tabla 7. Probabilidad de la mayoría de edad según el estadio $\mathrm{H}$ reportados en varias poblaciones.

\begin{tabular}{|c|c|c|c|}
\hline \multirow[b]{2}{*}{ País } & \multirow[b]{2}{*}{ Pieza } & \multicolumn{2}{|c|}{ Sexo } \\
\hline & & Femenino & Masculino \\
\hline Perú, Quezada & 38 & $100 \%$ & $99,2 \%$ \\
\hline Perú, Quezada & 48 & $100 \%$ & $99,1 \%$ \\
\hline Japon $^{22}$ & 38 & $99 \%$ & $98 \%$ \\
\hline Japon $^{22}$ & 48 & $99 \%$ & $98 \%$ \\
\hline Italia $^{19}$ & 38 & $98 \%$ & $96 \%$ \\
\hline Portugal $^{21}$ & 38 & $97,6 \%$ & $97,5 \%$ \\
\hline Portugal $^{21}$ & 48 & $98 \%$ & $98,1 \%$ \\
\hline Egipto $^{24}$ & 38 & $100 \%$ & $97,4 \%$ \\
\hline Egipto $^{24}$ & 48 & $100 \%$ & $100 \%$ \\
\hline EEUU $^{12 *}$ & 38 & $91 \%$ & $89 \%$ \\
\hline $\mathrm{EEUU}^{12 *}$ & 48 & $92 \%$ & $85 \%$ \\
\hline España $^{14}$ & 38 & $91,4 \%$ & $91,31 \%$ \\
\hline España $^{14}$ & 48 & $85,4 \%$ & $91,67 \%$ \\
\hline EEUU $^{17^{*}}$ & 38 & $89,9 \%$ & $85,8 \%$ \\
\hline $\mathrm{EEUU}^{17^{*}}$ & 48 & $87,1 \%$ & $86,4 \%$ \\
\hline EEUU $^{30}$ & 38 ó 48 & $92,2 \%$ & $90,1 \%$ \\
\hline EEUU $^{23}$ & 38 ó 48 & $93,4 \%$ & $89,9 \%$ \\
\hline EEUU $^{23}$ & 38 ó 48 & $84,0 \%$ & $92,7 \%$ \\
\hline Malasia $^{31}$ & 38 & $100 \%$ & $100 \%$ \\
\hline Malasia $^{31}$ & 48 & $99,5 \%$ & $100 \%$ \\
\hline Brasil $^{32}$ & 38 & $98 \%$ & $98 \%$ \\
\hline Brasil $^{32}$ & 48 & $98 \%$ & $98 \%$ \\
\hline
\end{tabular}

*Población de Hispanos

Solari y col. (12) encontraron en una población hispana en Texas, que aproximadamente el 91\% de mujeres y $85 \%$ de hombres, pueden tener como mínimo 18 años de edad, si el tercer molar se encuentra en estadio H. Porcentajes aún menores fueron encontrados por Kasper y col. (17) en una población hispana en Estados Unidos de Norteamérica en donde el $87,1 \%$ fueron mujeres y el $85,7 \%$ hombres (Tabla 7 ).

En conclusión, aunque posiblemente la única herramienta disponible hablando en términos biológicos, para la estimación de la edad en el grupo de adultos jóvenes sea el desarrollo y la mineralización del tercer molar; ésta debe ser usada con precaución con fines legales y forenses, debido a la variabilidad entre individuos por raza y sexo, ya que existe un rango de edad dentro de cada estadio de Demirjian.

\section{Correspondencia:}

Milushka Miroslava Quezada Márquez

Av. Honorio Delgado 430 - Lima 31, Perú.

Correo electrónico: milushka.quezada@upch.pe

\section{REFERENCIAS BIBLIOGRÁFICAS}

1. Schmeling A, Grundmann C, Fuhrmann A, et al. Criteria for age estimation in living individuals. Int $\mathrm{J}$ Legal Med. 2008; 122(6):457-60.

2. Olze A, Schmeling A, Taniguchi M, et al. Forensic age estimation in living subjects: the ethnic factor in wisdom tooth mineralization. Int J Legal Med. 2004; 118(3):1703.

3. Panchbhai AS. Dental radiographic indicators, a key to age estimation. Dentomaxillofac Radiol. 2011; 40(4): 199-212.

4. Knell B, Ruhstaller P, Prieels F, Schmeling A. Dental age diagnostics by means of radiographical evaluation of the growth stages of lower wisdom teeth. Int J Legal Med. 2009; 123(6):465-9.

5. Moorrees CF, Fanning EA, Hunt EE. Age variation of formation stages for ten permanent teeth. J Dent Res. 1963; 42(6):1490-502.

6. Nolla CM. Development of the permanent teeth. ASDC J Dent Child. 1960; 27(4):254-66.

7. Demirjian A, Goldstein H, Tanner JM. A new system of dental age assessment. Hum Biol. 1973; 45(2):211-27.

8. Gustafson G, Koch G. Age estimation up to 16 years of age based on dental development. Odontol Revy. 1974; 25(3):297-306.

9. Gleiser I, Hunt EE Jr. The permanent mandibular first molar: its calcification, eruption and decay. Am J Phys Anthropol. 1955; 13(2):253-83.

10. Harris MJ, Nortjé CJ. The mesial root of the third mandibular molar. A possible indicator of age J Forensic. Odontostomatol. 1984; 2(2):39-43.

11. Kullman L, Johanson G, Akesson L. Root development of the lower third molar and its relation to chronological age. Swed Dent J. 1992; 16(4):161-7.

12. Solari AC, Abramovitch K. The accuracy and precision of third molar development as an indicator of chronological age in Hispanics. J Forensic Sci. 2002; 47(3):531-5.

13. Olze A, Bilang D, Schmidt S, Wernecke KD, Geserick G, Schmeling A. Validation of common classification systems for assessing the mineralization of third molars. Int J Legal Med. 2005; 119(1):22-6.

14. Prieto JL, Barbería E, Ortega R, Magaña C. Evaluation of chronological age based on third molar development in the Spanish population. Int J Legal Med. 2005; 119(6):349-54.

15.Zeng DL, Wu ZL, Cui MY. Chronological age estimation of third molar mineralization of Han in southern China. Int J Legal Med. 2010; 124(2):119-23.

16. Araújo AM, Pontual ML, França K, Beltrão R, Pontual 
A. Association between mineralization of third molars and chronological age in a Brazilian sample. Rev Odonto Ciênc. 2010; 25(4):391-4.

17. Kasper KA, Austin D, Kvanli AH, Rios TR, Senn DR. Reliability of third molar development for age estimation in a Texas Hispanic population: a comparison study. J Forensic Sci. 2009; 54(3):651-7.

18. Rai B, Kaur J, Jafarzadeh H. Dental age estimation from the developmental stage of the third molars in Iranian population. J ForensicLegMed.2010;17(6):30911.

19. Cameriere R, Ferrante L, De Angelis D, Scarpino F, Galli F. The comparison between measurement of open apices of third molars and Demirjian stages to test chronological age of over 18 year olds in living subjects. Int J Legal Med. 2008; 122(6):493-7.

20. Cantekin K, Yilmaz Y, Demirci T, Celikoglu M. Morphologic analysis of third-molar mineralization for eastern Turkish children and youth. J Forensic Sci. 2012; 57(2):531-4.

21. Caldas IM, Júlio P, Simões RJ, Matos E, Afonso A, Magalhães T. Chronological age estimation based on third molar development in a Portuguese population. Int J Legal Med. 2011; 125(2):235-43.

22. Arany S, Iino M, Yoshioka N. Radiographic survey of third molar development in relation to chronological age among Japanese juveniles. J Forensic Sci. 2004; 49(3):534-8.

23. Blankenship JA, Mincer HH, Anderson KM, Woods MA, Burton EL. Third molar development in the estimation of chronologic age in american blacks as compared with whites. J Forensic Sci. 2007; 52(2):42833.

24. Hassan NA, Abo N. Orthopantomography and age determination using third molar mineralization in a sample of egyptians. J Forensic Med Clin Toxicol. 2007; 15 (1):45-58.
25. Verma P, Sachdeo J, Verma KG, Gupta SD, Guruprasad R. Age estimation of adolescents and young adults based on development of mandibular third molars: A panoramic study. J Indian Acad Oral Med Rad. 2011; 23(1):9-13.

26. Boonpitaksathit T, Hunt N, Roberts GJ, Petrie A, Lucas VS. Dental age assessment of adolescents and emerging adults in United Kingdom Caucasians using censored data for stage $\mathrm{H}$ of third molar roots. Eur J Orthod. 2011; 33(5):503-8.

27. Sisman Y, Uysal T, Yagmur F, Ramoglu SI. Third-molar development in relation to chronologic age in Turkish children and young adults. Angle Orthod. 2007; 77(6):1040-5.

28. Olze A, Pynn BR, Kraul V, et al. Studies on the chronology of third molar mineralization in First Nations people of Canada. Int J Legal Med. 2010; 124(5):433-7.

29. Li G, Ren J, Zhao S, et al. Dental age estimation from the developmental stage of the third molars in western Chinese population. Forensic Sci Int. 2012; 219(10):15864.

30. Mincer HH, Harris EF, Berryman HE. The A.B.F.O. study of third molar development and its use as an estimator of chronological age. J Forensic Sci. 1993; 38(2):379-90.

31. Johan NA, Khamis MF, Abdul Jamal NS, Ahmad B, Mahanani ES. The variability of lower third molar development in Northeast Malaysian population with application to age estimation. $\mathrm{J}$ Forensic Odontostomatol. 2012; 30(1):45-54.

32. de Oliveira FT, Capelozza AL, Lauris JR, de Bullen IR. Mineralization of mandibular third molars can estimate chronological age Brazilian indices. Forensic Sci Int. 2012; 219(10):147-50.

Recibido: $14 / 06 / 2013$

Aceptado: 11/10/2013 